\title{
Fundamental limit to single-atom analysis by STEM-EDX spectroscopy
}

\author{
M. Watanabe ${ }^{1}$ and R.F. Egerton ${ }^{2}$. \\ 1. Dept of Materials Science and Engineering, Lehigh University, Bethlehem. PA 18015. \\ 2. Physics Department, University of Alberta, Edmonton, Canada T6G 2E1.
}

Within the last few years, single-atom catalysts, consisting of single metal atoms on a metal-oxide support, have been synthesized and shown to offer high efficiency [1-3]. Analytical electron microscopy in the scanning transmission electron microscope (STEM) provides a means of identifying individual atoms at known locations on the substrate. However such measurements are difficult, so the experimental conditions need to be optimized. We present below some results of calculations that indicate that an important parameter is the microscope accelerating voltage, which should be chosen to be as low as possible, consistent with adequate resolution.

This conclusion stems from the possibility of knock-on displacement of the atom along the surface or into the vacuum of the microscope. The average number of atom displacements within a recording time $\mathrm{T}$ is $\mathrm{N}_{\mathrm{d}}=\sigma_{\mathrm{d}}(\mathrm{J} \mathrm{T} / \mathrm{e})=\sigma_{\mathrm{d}} \mathrm{D}_{\mathrm{e}}$ where $\sigma_{\mathrm{d}}$ is a displacement cross section, $\mathrm{J}$ is the current density in the electron probe and $D_{e}$ is the electron dose (particles per unit area). Setting $N_{d}=1$ gives $D_{e}=1 / \sigma_{d}$, setting a limit to the number of characteristic x-ray photons that can be used to identify the metal atom.

The average number of such photons is $N_{x}=\sigma_{x}$ De where $\sigma_{x}$ is a cross section for $x$-ray detection, which we evaluate as $\sigma_{\mathrm{k}} \omega_{\mathrm{k}} \eta, \sigma_{\mathrm{k}}$ being the ionization cross section of atomic shell $\mathrm{k}, \omega_{\mathrm{k}}$ is the corresponding fluorescence yield and $\eta$ is the $x$-ray collection efficiency (which we take as 0.1 , assuming a large-area detector with solid angle $\sim 1$ sterad). With $D_{\mathrm{e}}=1 / \sigma_{\mathrm{d}}$, we have: $\mathrm{N}_{\mathrm{x}}=\sigma_{\mathrm{x}} / \sigma_{\mathrm{d}}$ and under conditions under which knock-on displacement of surface atoms is possible, $\sigma_{\mathrm{d}}$ can easily exceed $\sigma_{\mathrm{x}}$, giving $\mathrm{N}_{\mathrm{x}}<1$ (see Fig. 2). Reliable elemental identification is then impossible.

Atomic displacement is avoided if the binding energy of the atom $E_{d}$ exceeds the maximum energy transfer $E_{\max }$ from the primary electron, whose value is given by relativistic kinematics [4]. Detectability of the peak is then determined by its signal/noise ratio, for example using the Rose criterion for a spectrum (SNR > 3). The Poisson statistical noise is approximately $\left(\mathrm{N}_{\mathrm{x}}+\mathrm{Nb}_{\mathrm{b}}\right)^{1 / 2}\left(\mathrm{~N}_{\mathrm{b}}\right.$ is the background count), giving $S N R=N_{x} /\left(N_{x}+N_{b}\right)^{1 / 2}$, which has been evaluated in Fig. 2 by taking $D_{e}=6 \times 10^{27} \mathrm{e} / \mathrm{m}^{2}$ (for example $0.1 \mathrm{nA}$ probe current, $0.3 \mathrm{~nm}$ probe diameter, $\mathrm{T}=1 \mathrm{~s}$ dwell time). In this example, the Ni atom would just be identifiable (SNR 3). The background count can be included as $\mathrm{N}_{b}=\mathrm{N}_{\mathrm{s}} \sigma_{b} \eta_{\mathrm{b}} \mathrm{D}_{\mathrm{e}}$, where $\sigma_{b}$ is a bremstrahlung cross section [5] and $N_{s}$ is the number of substrate atoms within the beam. Although most of the bremstrahlen come from the substrate (assumed to contain $\mathrm{N}_{\mathrm{s}} \sim 60$ atoms of mean atomic number 10), $\mathrm{N}_{\mathrm{b}}<<\mathrm{N}_{\mathrm{x}}$ for a reasonable thin support and a small-diameter probe.

The value of $E_{d}$ will depend on the atomic site of the atom, so some atoms may be detectable and others not. Ejection may be less efficient if the atom lies on the beam-entrance surface of the specimen. $E_{d}$ is certainly lower for momentum transfer along the surface (adatom motion rather than surface sputtering) but the maximum energy available is a factor or $2-4$ lower [6]. In all cases, $E_{\max }$ is roughly proportional to the incident energy $\mathrm{E}_{0}$, so use of a low beam energy could be important. Recent EDXS identifications of single Er [7] and Pt [8] atoms used an accelerating voltage of $60 \mathrm{kV}$. EELS may be a preferable technique for identifying certain elements due to its greater signal-collection efficiency [9]. 


\section{References:}

[1] Q. Fu, H. Saltsburg, M. Flytzani-Stephanopoulos, Science 301 (2003), p. 935.

[2] M Yang et al., J. Am. Chem. Soc. 135 (2013), p. 3768.

[3] LF Allard, S. Duan and J Liu, Microsc. Microanal. 22 Suppl. 3 (2016), p.876.

[4] RF Egerton et al., Ultramicroscopy 110 (2010), p. 991.

[5] SM Seltzer and M.J. Berger, Atomic Data \& Nuclear Data Tables 35 (1986), p. 345.

[6] RF Egerton, Microsc. Microanal. 19 (2013), p. 479.

[7] K Suenaga, et al., Nature Photonics 6 (2012) p.545.

[8] T Lovejoy et al., Appl.Phys. Lett. 100 (2012), 154101.

[9] MW wishes to acknowledge financial support from the NSF through grants DMR-0804528 and DMR-1040229. RFE is grateful to NSERC for funding.

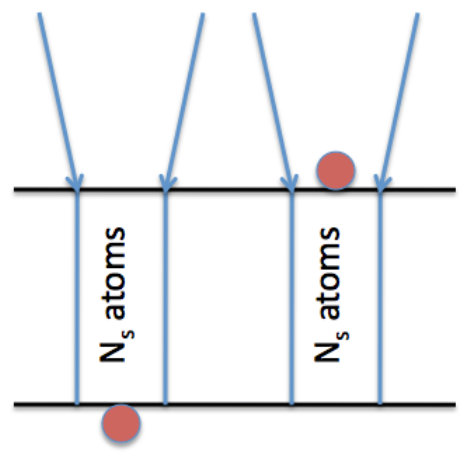

(a)

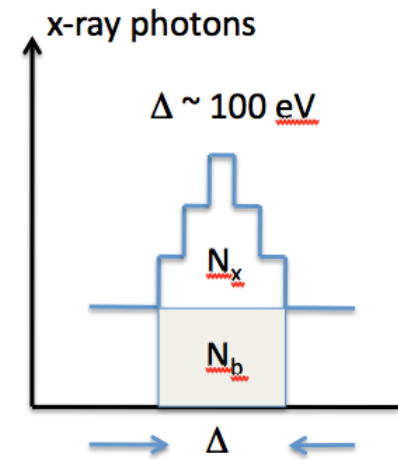

(b)

Figure 1. (a) Metal atoms on the bottom and top surface of a thin support. (b) Characteristic x-ray peak and its background.

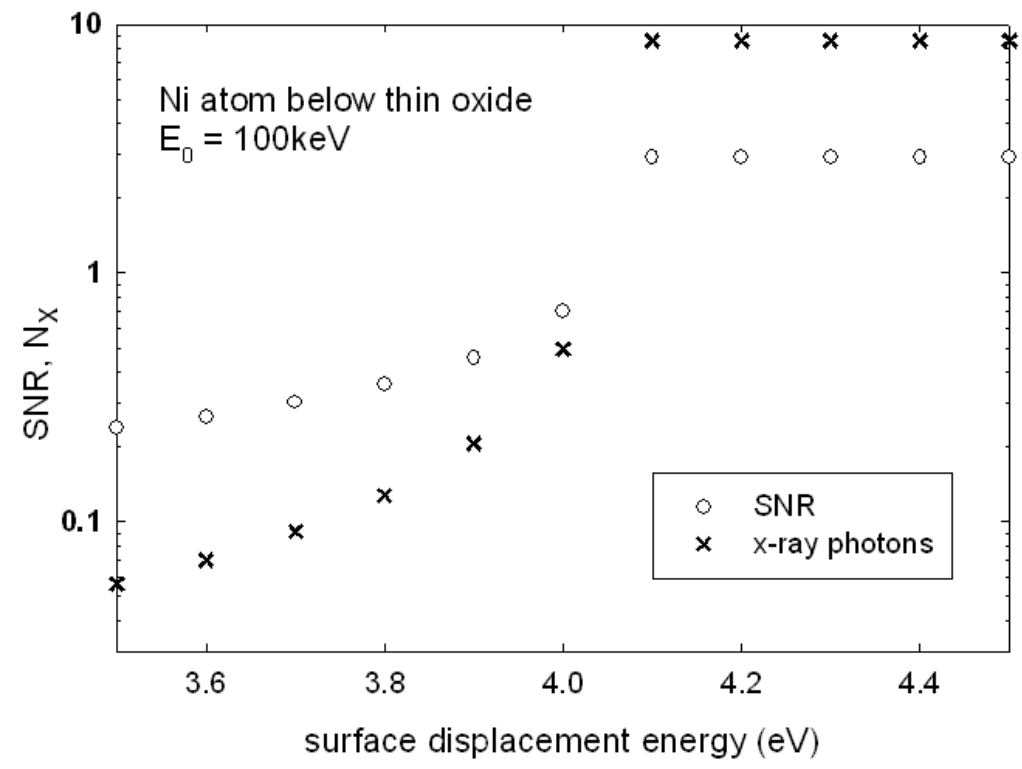

Figure 2. Number $\mathrm{N}_{\mathrm{x}}$ of $\mathrm{x}$-ray photons emitted by a single $\mathrm{Ni}$ atom and signal/noise ratio of the characteristic $\mathrm{K}$-emission peak, as a function of the atomic binding energy $\mathrm{E}_{\mathrm{d}}$, the atom assumed to lie on the beam-exit surface of the specimen. For $\mathrm{E}_{\mathrm{d}}>\mathrm{E}_{\max } \sim 4.1 \mathrm{eV}$, we assume an atom dwell time of $\mathrm{T}=$ $1 \mathrm{~s}$ and current density $10^{5} \mathrm{~A} / \mathrm{cm}^{2}$ (e.g. $0.1 \mathrm{nA}$ probe current, $0.3 \mathrm{~nm}$ probe diameter). 